\title{
Measuring the scalar induced gravitational waves from observations
}

\author{
Jun $\mathbf{L i}^{1,2, a}$, Guang-Hai Guo ${ }^{1, b}$ \\ ${ }^{1}$ School of Mathematics and Physics, Qingdao University of Science and Technology, Qingdao 266061, China \\ ${ }^{2}$ CAS Key Laboratory of Theoretical Physics, Institute of Theoretical Physics, Chinese Academy of Sciences, Beijing 100190, China
}

Received: 25 January 2021 / Accepted: 1 July 2021 / Published online: 12 July 2021

(c) The Author(s) 2021

\begin{abstract}
We consider the scalar induced gravitational waves from the cosmic microwave background (CMB) observations and the gravitational wave observations. In the $\Lambda \mathrm{CDM}+r$ model, we constrain the cosmological parameters within the evolution of the scalar induced gravitational waves by the additional scalar power spectrum. The two special cases called narrow power spectrum and wide power spectrum have influence on the cosmological parameters, especially the combinations of Planck18+BAO+BK15+LISA. We also compare these numerical results from four datasets within LIGO, LISA, IPTA and FAST projects, respectively. The constraints from FAST have a significant impact on tensor-to-scalar ratio. Besides, we only consider the relic density of induced gravitational waves with respect to different frequencies from $\mathrm{CMB}$ scale to high frequencies including the range of LIGO and LISA.
\end{abstract}

\section{Introduction}

Modern cosmology is based on general relativity and the premise that the Universe is homogeneous isotropic on large scale. On this background, the early Universe was highly inhomogeneous on small scale and the perturbations would evolve into stars, galaxies and galaxy clusters over time. The cosmological linear perturbation theory has been developed so fast these decades owning to the cosmological observations, such as the cosmic microwave background (CMB). The theoretical first-order approximation from perturbations has been revealed through the $\mathrm{CMB}$ observations which include the Wilkinson Microwave Anisotropy Probe (WMAP) [1], the Planck satellite [2], the BICEP2 and Keck array (BK) [3], and the Baryon Acoustic Oscillation (BAO) [4-6]. Depend on the cosmological linear perturbation theory and CMB obser-

\footnotetext{
a e-mail: lijun@qust.edu.cn (corresponding author)

be-mail: ghguo@qust.edu.cn
}

vations, the standard cosmological model has been established and corresponding cosmological parameters are measured accurately.

However, the rapid developments of the cosmological observations inspire us considering the deviation from the first-order approximation. The second-order cosmological perturbation theory has been proposed in literatures [7]. The result shows that the curvature perturbations couple to the tensor perturbations at second-order which produce the scalar induced gravitational waves in the radiation dominated era. The power spectrum from the curvature perturbations influences the evolution of the scalar induced gravitational waves. We should consider the scalar induced gravitational waves from theoretical and observational sides. Applications of the second-order cosmological perturbation theory to the analysis of the cosmological observations become significant. See some other related works in [8-33]. The gauge dependence or independence of induced gravitational waves are discussed in these papers [11-16]. The induced gravitational waves in different cosmological backgrounds are explored in [17-25]. The induced gravitational waves not only probe the primordial density perturbation spectrum on small scales, but also probe the thermal history of the universe [28-30].

In this paper, we add the scalar power spectrum as presented in a previous study [8] and measure the scalar induced gravitational waves from the CMB observations and the gravitational wave observations. Here, the observations include the Planck satellite, the BICEP2 and Keck array, the Baryon Acoustic Oscillation, the Laser Interferometer Gravitationalwave Observatory (LIGO) detector [34-36], the Laser Interferometer Space Antenna (LISA) detector [36,37] and two Pulsar Timing Array projects [38-41], namely the International Pulsar Timing Array (IPTA) [39] and the Fivehundred-meter Aperture Spherical radio Telescope (FAST) [40]. The data of Advanced LIGO are from the second observing run $(\mathrm{O} 2)$ and first observing run $(\mathrm{O} 1)$ in the high frequency from 20 to $1726 \mathrm{~Hz}$. The detect ability of LISA 
is in the frequency from $10^{-4}$ to $1 \mathrm{~Hz}$. The detect ability of IPTA is in the low frequency from $1.58 \times 10^{-9}$ to $8.27 \times 10^{-7} \mathrm{~Hz}$ and the detect ability of FAST is in the frequency from $6.34 \times 10^{-10}$ to $8.27 \times 10^{-7} \mathrm{~Hz}$. The gravitational wave observations are taken as our previous works $[42,43]$.

\section{The power spectrum and the scalar induced gravitational waves}

The second-order perturbation metric about the FriedmannRobert-Walker background in the conformal Newtonian gauge is taken as [25-27]

$d s^{2}=a^{2}\left\{-(1+2 \phi) d \eta^{2}+\left[(1-2 \phi) \delta_{i j}+\frac{h_{i j}}{2}\right] d x^{i} d x^{j}\right\}$,

Table 1 The $68 \%$ limits on the cosmological parameters in the $\Lambda \mathrm{CDM}+r$ model from the combinations of Planck18+BAO+BK15 $+\mathrm{LIGO}$, Planck18+BAO+BK15+LIGO ${ }^{n}$, Planck18+BAO+BK15+LIGO $^{w}$ where $\eta$ is the conformal time, $\phi$ is the scalar perturbation and $h_{i j}$ is the gravitational wave perturbation.

In the radiation dominated Universe, the fraction of the gravitational wave energy density per logarithmic wavelength is given by $[25,26]$

$\Omega_{\mathrm{GW}}(\eta, k)=\frac{1}{24}\left(\frac{k}{a(\eta) H(\eta)}\right)^{2} \overline{P_{h}(\eta, k)}$,

where $P_{h}(\eta, k)$ is the power spectrum of the induced gravitational waves

$$
P_{h}(\eta, k)=4 \int_{0}^{\infty} \mathrm{d} v \int_{|1-v|}^{1+v} \mathrm{~d} u f^{2}(v, u, x) P_{\zeta}(k v) P_{\zeta}(k u),(3)
$$

and $P_{\zeta}(k)$ is the power spectrum of the primordial curvature perturbations. The function $f(v, u, x)$ is defined as

$$
f(v, u, x)=I(v, u, x) \frac{4 v^{2}-\left(1+v^{2}-u^{2}\right)^{2}}{4 v u},
$$

where function $I(v, u, x)$ comes from the source term.

and the combinations of Planck18+BAO+BK15+LISA, Planck18+BAO+BK15+LISA ${ }^{n}, \quad$ Planck18+BAO+BK15+LISA ${ }^{w}$,

\begin{tabular}{|c|c|c|c|c|c|c|}
\hline Parameters & $\begin{array}{l}\text { Planck18+BAO } \\
\text { +BK15+LIGO }\end{array}$ & $\begin{array}{l}\text { Planck18+BAO } \\
+ \text { +BK15+LIGO }\end{array}$ & $\begin{array}{l}\text { Planck18+BAO } \\
\quad+\text { BK15+LIGO }\end{array}$ & $\begin{array}{r}\text { Planck18+BAO } \\
+ \text { +BK15+LISA }\end{array}$ & $\begin{array}{l}\text { Planck18+BAO } \\
+ \text { BK15+LISA }{ }^{n}\end{array}$ & $\begin{array}{l}\text { Planck18+BAO } \\
+ \text { +BK15+LISA }\end{array}$ \\
\hline$\Omega_{b} h^{2}$ & $0.02240_{-0.00013}^{+0.00014}$ & $\begin{array}{c}0.02240 \pm \\
0.00013\end{array}$ & $\begin{array}{c}0.02240 \pm \\
0.00013\end{array}$ & $\begin{array}{c}0.02240 \pm \\
0.00013\end{array}$ & $\begin{array}{c}0.02240 \pm \\
0.00013\end{array}$ & $\begin{array}{c}0.02240 \pm \\
0.00013\end{array}$ \\
\hline$\Omega_{c} h^{2}$ & $\begin{array}{c}0.11958 \pm \\
0.00096\end{array}$ & $\begin{array}{c}0.11956 \pm \\
0.00094\end{array}$ & $\begin{array}{c}0.11956 \pm \\
0.00094\end{array}$ & $0.11959_{-0.00095}^{+0.00094}$ & $\begin{array}{c}0.11954 \pm \\
0.00093\end{array}$ & $0.11957_{-0.00095}^{+0.00094}$ \\
\hline $100 \theta_{\mathrm{MC}}$ & $\begin{array}{c}1.04099 \pm \\
0.00029\end{array}$ & $1.04098_{-0.00029}^{+0.00030}$ & $\begin{array}{c}1.04099 \pm \\
0.00029\end{array}$ & $\begin{array}{c}1.04099 \pm \\
0.00029\end{array}$ & $\begin{array}{c}1.04099 \pm \\
0.00029\end{array}$ & $\begin{array}{c}1.04099 \pm \\
0.00029\end{array}$ \\
\hline$\tau$ & $0.0567_{-0.0078}^{+0.0069}$ & $0.0568_{-0.0078}^{+0.0070}$ & $0.0568_{-0.0076}^{+0.0069}$ & $0.0567_{-0.0076}^{+0.0069}$ & $0.0569_{-0.0077}^{+0.0069}$ & $0.0567_{-0.0077}^{+0.0069}$ \\
\hline $\ln \left(10^{10} A_{s}\right)$ & $3.049_{-0.015}^{+0.014}$ & $3.049 \pm 0.014$ & $3.049 \pm 0.014$ & $3.049_{-0.015}^{+0.014}$ & $3.049 \pm 0.014$ & $3.049 \pm 0.014$ \\
\hline$n_{s}$ & $0.9654 \pm 0.0039$ & $0.9654 \pm 0.0038$ & $0.9655 \pm 0.0038$ & $0.9654_{-0.0038}^{+0.0037}$ & $0.9655 \pm 0.0037$ & $0.9655 \pm 0.0038$ \\
\hline$r_{0.05}(95 \% \mathrm{CL})$ & $<0.076$ & $<0.075$ & $<0.074$ & $<0.075$ & $<0.075$ & $<0.073$ \\
\hline
\end{tabular}
respectively
Table 2 The $68 \%$ limits on the cosmological parameters in the $\Lambda \mathrm{CDM}+r$ model from the combinations of Planck18+BAO+BK15+IPTA,
Planck18+BAO+BK15+IPTA $^{w}$ and the combinations of Planck18+BAO+BK15+FAST, Planck18+BAO+BK15+FAST ${ }^{n}$,

\begin{tabular}{|c|c|c|c|c|c|c|}
\hline Parameters & $\begin{array}{r}\text { Planck18+BAO } \\
\text { +BK15+IPTA }\end{array}$ & $\begin{array}{l}\text { Planck18+BAO } \\
\quad+\text { BK15+IPTA }\end{array}$ & $\begin{array}{c}\text { Planck18+BAO+ } \\
\text { BK15+IPTA }^{w}\end{array}$ & $\begin{array}{c}\text { Planck18+BAO+ } \\
\text { BK15+FAST }\end{array}$ & $\begin{array}{l}\text { Planck18+BAO } \\
+ \text { +BK15+FAST }\end{array}$ & $\begin{array}{l}\text { Planck18+BAO } \\
+ \text { +BK15+FAST }\end{array}$ \\
\hline$\Omega_{b} h^{2}$ & $\begin{array}{c}0.02241 \pm \\
0.00013\end{array}$ & $\begin{array}{c}0.02240 \pm \\
0.00013\end{array}$ & $\begin{array}{c}0.02240 \pm \\
0.00013\end{array}$ & $\begin{array}{c}0.02240 \pm \\
0.00013\end{array}$ & $\begin{array}{c}0.02240 \pm \\
0.00013\end{array}$ & $\begin{array}{c}0.02240 \pm \\
0.00014\end{array}$ \\
\hline$\Omega_{c} h^{2}$ & $\begin{array}{c}0.11957 \pm \\
0.00093\end{array}$ & $0.11957_{-0.00095}^{+0.00094}$ & $0.11957_{-0.00093}^{+0.00095}$ & $\begin{array}{c}0.11958 \pm \\
0.00094\end{array}$ & $\begin{array}{c}0.11959 \pm \\
0.00094\end{array}$ & $\begin{array}{c}0.11960 \pm \\
0.00094\end{array}$ \\
\hline $100 \theta_{\mathrm{MC}}$ & $\begin{array}{c}1.04099 \pm \\
0.00029\end{array}$ & $\begin{array}{c}1.04099 \pm \\
0.00029\end{array}$ & $\begin{array}{c}1.04099 \pm \\
0.00030\end{array}$ & $\begin{array}{c}1.04099 \pm \\
0.00029\end{array}$ & $\begin{array}{c}1.04098 \pm \\
0.00029\end{array}$ & $\begin{array}{c}1.04099 \pm \\
0.00029\end{array}$ \\
\hline$\tau$ & $0.0568_{-0.0077}^{+0.0069}$ & $0.0568_{-0.0078}^{+0.0069}$ & $0.0568_{-0.0078}^{+0.0071}$ & $0.0569_{-0.0078}^{+0.0070}$ & $0.0570_{-0.0078}^{+0.0071}$ & $0.0569_{-0.0077}^{+0.0069}$ \\
\hline $\ln \left(10^{10} A_{s}\right)$ & $3.049 \pm 0.014$ & $3.049_{-0.015}^{+0.014}$ & $3.049 \pm 0.014$ & $3.049_{-0.015}^{+0.014}$ & $3.050_{-0.015}^{+0.014}$ & $3.049 \pm 0.014$ \\
\hline$n_{s}$ & $0.9655 \pm 0.0037$ & $0.9654 \pm 0.0038$ & $0.9655_{-0.0038}^{+0.0037}$ & $0.9654 \pm 0.0038$ & $0.9653 \pm 0.0038$ & $0.9653 \pm 0.0037$ \\
\hline$r_{0.05}(95 \% \mathrm{CL})$ & $<0.075$ & $<0.075$ & $<0.075$ & $<0.049$ & $<0.049$ & $<0.049$ \\
\hline
\end{tabular}
Planck18+BAO+BK15+FAST $^{w}$, respectively 
Here, we consider adding the scalar power spectrum which is peaked at $k_{*}$ and parameterized as [8]

$P_{\zeta}(k)= \begin{cases}\frac{k-k_{-}}{k_{*}-k_{-}}, & \text {for } k_{-}<k<k_{*}, \\ \frac{k_{+}-k}{k_{+}-k_{*}}, & \text { for } k_{*}<k<k_{+},\end{cases}$

where choose $k_{*}=10^{15} \mathrm{Mpc}^{-1}$ with the same reason as our previous work [44]. The amplitude of the additional scalar power spectrum is much larger than those at CMB scales which provide a new way to probe primordial black holes. We also introduce the parameter $\Delta$ to quantify the width of the additional scalar power spectrum

$$
\Delta=\frac{k_{+}-k_{-}}{k_{*}},
$$

and discuss two simple examples. The narrow power spectrum with $k_{-}=0.995 k_{*}$ and $k_{+}=1.005 k_{*}$ takes the form

$$
P_{\zeta}(k)= \begin{cases}\frac{k-0.995 \times 10^{15}}{0.005 \times 10^{15}}, & \text { for } 0.995 \times 10^{15}<k<10^{15} \\ \frac{1.005 \times 10^{15}-k}{0.005 \times 10^{15}}, & \text { for } 10^{15}<k<1.005 \times 10^{15}\end{cases}
$$

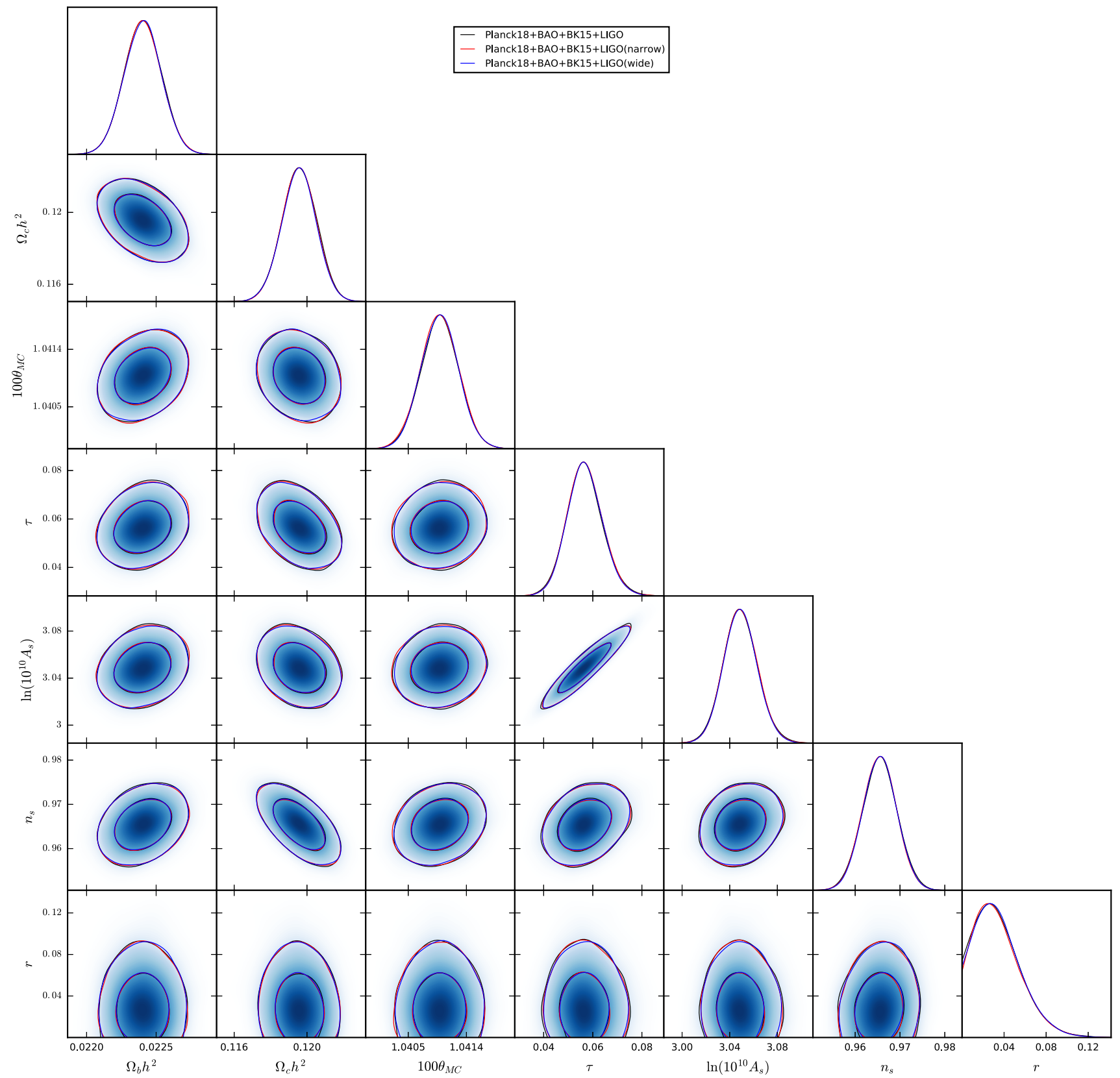

Fig. 1 The contour plots and the likelihood distributions for the cosmological parameters in the $\Lambda$ CDM $+r$ model at the $68 \%$ and $95 \%$ CL from the combinations of Planck18+BAO+BK15+LIGO, Planck18+BAO+BK15+LIGO ${ }^{n}$, Planck18+BAO+BK15+LIGO ${ }^{w}$, respectively 
The wide power spectrum with $k_{-}=0.5 k_{*}$ and $k_{+}=10.5 k_{*}$ takes the form

$P_{\zeta}(k)= \begin{cases}\frac{k-0.5 \times 10^{15}}{0.5 \times 10^{15}}, & \text { for } 0.5 \times 10^{15}<k<10^{15}, \\ \frac{10.5 \times 10^{15}-k}{9.5 \times 10^{15}}, & \text { for } 10^{15}<k<10.5 \times 10^{15} .\end{cases}$

We compare Eq. (5) with the power spectrum of the scalar perturbations which parameterized as

$P_{S}(k)=A_{s}\left(\frac{k}{k_{+}}\right)^{n_{s}-1}$,

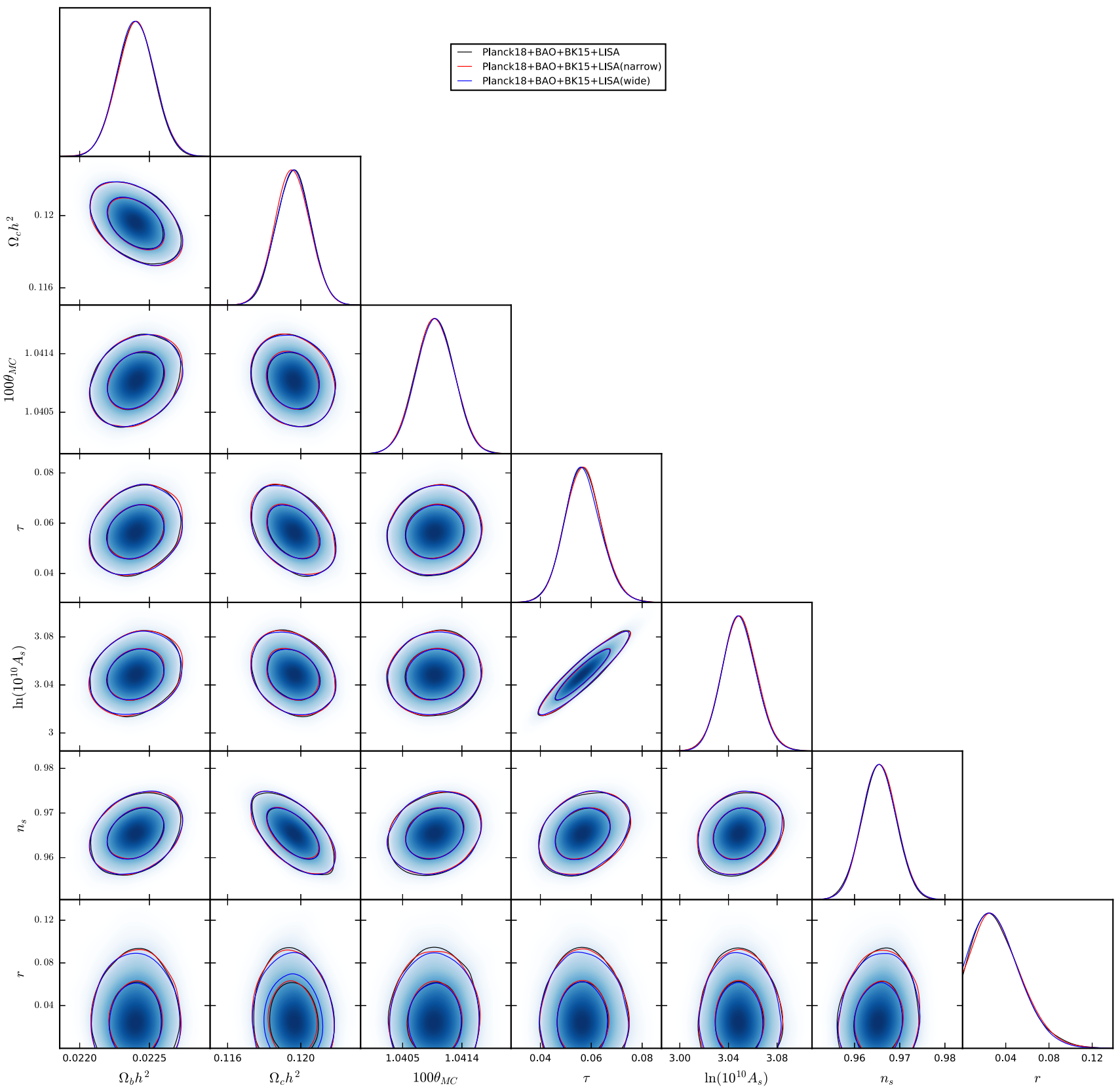

Fig. 2 The contour plots and the likelihood distributions for the cosmological parameters in the $\Lambda \mathrm{CDM}+r$ model at the $68 \%$ and $95 \% \mathrm{CL}$ from the combinations of Planck18+BAO+BK15+LISA, Planck18+BAO+BK15+LISA ${ }^{n}$, Planck18+BAO+BK15+LISA ${ }^{w}$, respectively 


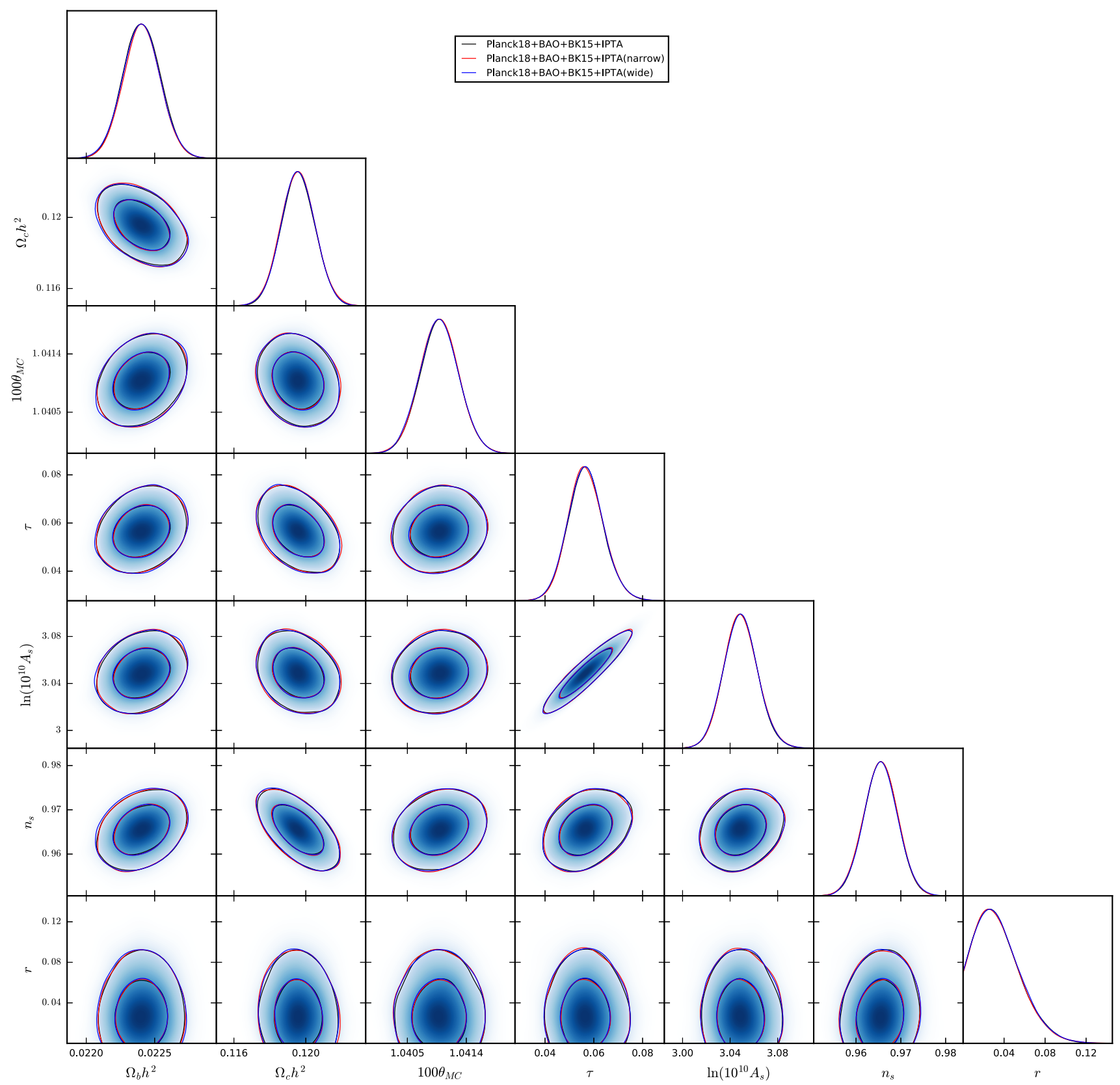

Fig. 3 The contour plots and the likelihood distributions for the cosmological parameters in the $\Lambda \mathrm{CDM}+r$ model at the $68 \%$ and $95 \%$ CL from the combinations of Planck18+BAO+BK15+IPTA, Planck18+BAO+BK15+IPTA ${ }^{n}$, Planck18+BAO+BK15+IPTA ${ }^{w}$, respectively

gravitational waves from the CMB observations and the gravitational wave observations as below.

\section{Constraints on the cosmological parameters}

We use the publicly available codes Cosmomc [45] to constrain the cosmological parameters within the evolution of the scalar induced gravitational waves by the additional scalar power spectrum. ${ }^{1}$ In the standard $\Lambda \mathrm{CDM}$ model, the six parameters are the baryon density parameter $\Omega_{b} h^{2}$, the cold dark matter density $\Omega_{c} h^{2}$, the angular size of the horizon at the last scattering surface $\theta_{\mathrm{MC}}$, the optical depth $\tau$, the scalar amplitude $A_{s}$ and the scalar spectral index $n_{s}$. We extend this

\footnotetext{
1 The routine for the second order cosmic perturbations and the induced gravitational waves in the Cosmomc code can be implemented by the power-tilt.f90 file and the cliklike.f90 file. The power-tilt.f90 file in the camb folder contains the information of the power spectra. The cliklike.f90 file in the source folder contains the information of the likelihood.
} 
model by adding the parameter $r$ and consider these seven parameters as fully free parameters. The parameter $r$, called tensor-to-scalar ratio, quantify the tensor amplitude $A_{t}$ compared to the scalar amplitude $A_{s}$ at the pivot scale

$r \equiv \frac{A_{t}}{A_{s}}$

The consistency relation is ignored for more inflation models. Our numerical results are given in Tables 1 and 2 and Figs. 1, $2,3,4,5$ and 6 .
In the $\Lambda \mathrm{CDM}+r$ model, the combinations of LIGO and Planck18+BAO+BK15 constrain the seven parameters accurately which have presented in [43]. When we consider the narrow power spectrum and the wide power spectrum as Eqs. (7) and (8), the corresponding numerical results of Planck18+BAO+BK15+LIGO ${ }^{n}$ and Planck18+BAO+BK15 $+\mathrm{LIGO}^{w}$ change slightly. The effects from the scalar induced gravitational waves on the cosmological parameters are not obvious. The constraint on parameter $r$ is

$r<0.076(95 \% \mathrm{CL})$

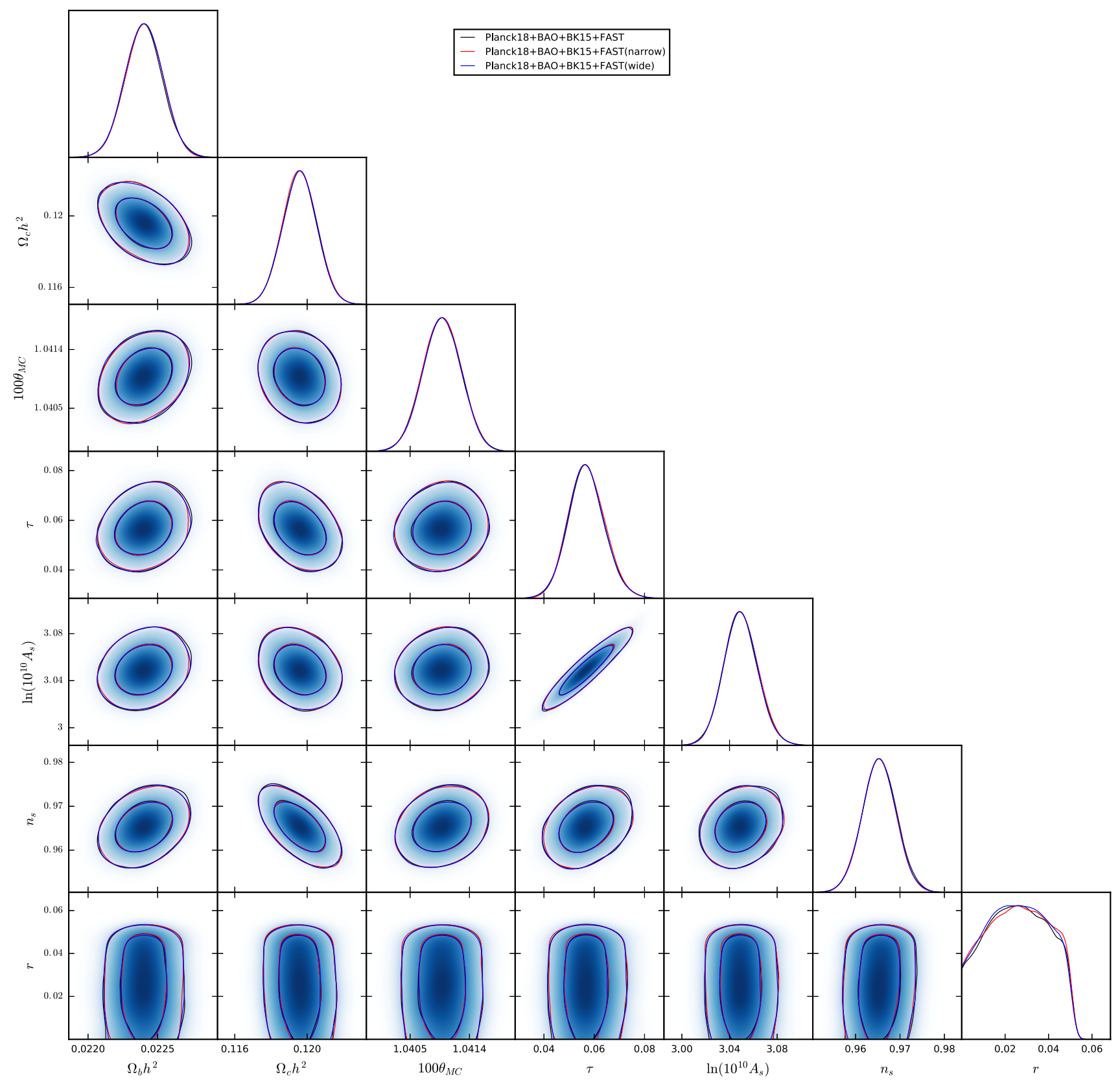

Fig. 4 The contour plots and the likelihood distributions for the cosmological parameters in the $\Lambda$ CDM+r model at the $68 \%$ and $95 \%$ CL from the combinations of Planck18+BAO+BK15+FAST, Planck18+BAO+BK15+FAST ${ }^{n}$, Planck18+BAO+BK15+FAST ${ }^{w}$, respectively 
from Planck18+BAO+BK15+LIGO datasets;

$r<0.075$ (95\% CL),

from Planck18+BAO+BK15+LIGO ${ }^{n}$ datasets;

$$
r<0.074 \quad(95 \% \mathrm{CL})
$$

from Planck18+BAO+BK15+LIGO ${ }^{w}$ datasets which are given in Table 1 and Fig. 1.

The combinations of Planck18+BAO+BK15+LISA ${ }^{n}$ and Planck18+BAO+BK15+LISA ${ }^{w}$ constrain the seven parame- ters better which is obvious from the contour plot of the cold dark matter density $\Omega_{c} h^{2}$ and the tensor-to-scalar ratio $r$ in Fig. 2. The constraint on parameter $r$ is

$r<0.075 \quad(95 \% \mathrm{CL})$

from Planck18+BAO+BK15+LISA datasets;

$r<0.075 \quad(95 \% \mathrm{CL})$

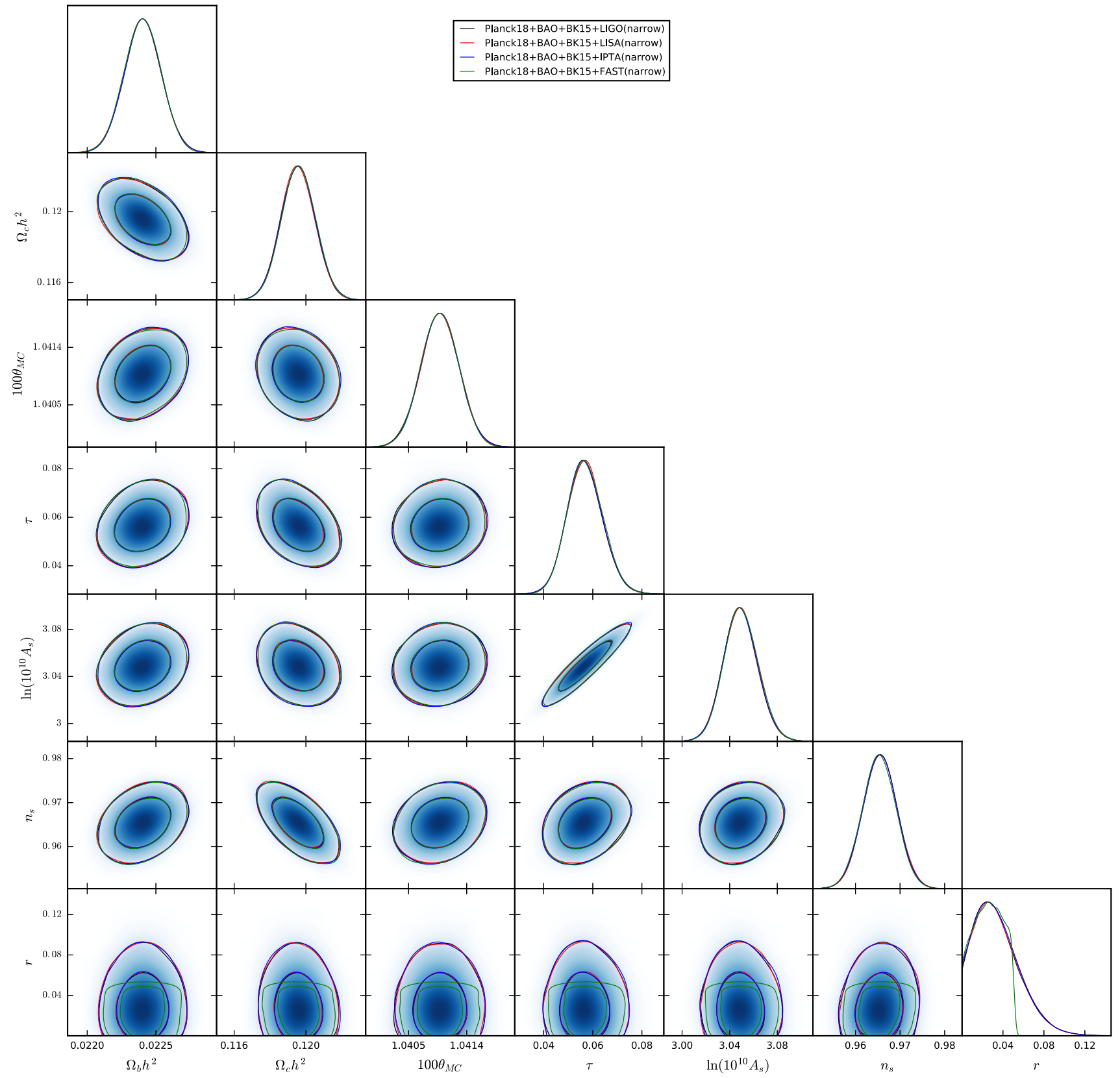

Fig. 5 The contour plots and the likelihood distributions for the cosmological parameters in the $\Lambda \mathrm{CDM}+r$ model at the $68 \%$ and $95 \% \mathrm{CL}$ from the combinations of Planck18+BAO+BK15+LIGO ${ }^{n}$, Planck18+BAO+BK15+LISA $^{n}$, Planck18+BAO+BK15+IPTA ${ }^{n}$, Planck18+BAO+BK15+FAST $^{n}$, respectively 
from Planck18+BAO+BK15+LISA ${ }^{n}$ datasets;

$r<0.073 \quad(95 \% \mathrm{CL})$,

from Planck18+BAO+BK15+LISA ${ }^{w}$ datasets.

The combinations of Planck18+BAO+BK15+IPTA ${ }^{n}$ and Planck18+BAO+BK15+IPTA ${ }^{w}$ effect the seven parameters slightly which is given in Table 2 and Fig. 3. The constraint on parameter $r$ is

$r<0.075 \quad(95 \% \mathrm{CL})$, from Planck18+BAO+BK15+IPTA datasets;

$r<0.075 \quad(95 \% \mathrm{CL})$

from Planck18+BAO+BK15+IPTA ${ }^{n}$ datasets;

$r<0.075 \quad(95 \% \mathrm{CL})$

from Planck18+BAO+BK15+IPTA ${ }^{w}$ datasets. The influence on the cosmological parameters from the additional scalar power spectrum are not obvious.
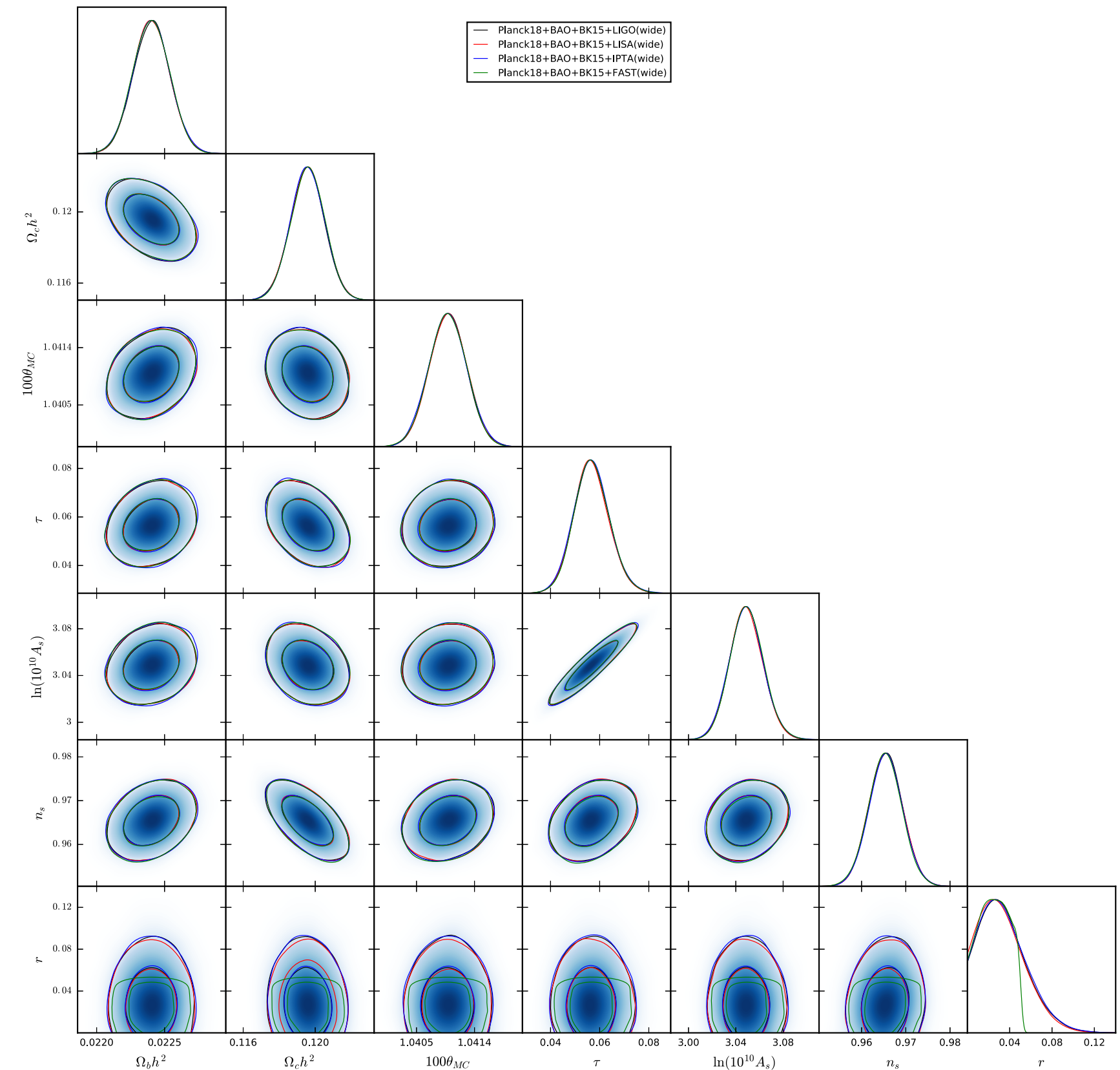

Fig. 6 The contour plots and the likelihood distributions for the cosmological parameters in the $\Lambda \mathrm{CDM}+r$ model at the $68 \%$ and $95 \% \mathrm{CL}$ from the combinations of Planck18+BAO+BK15+LIGO ${ }^{w}$, Planck18+BAO+BK15+LISA ${ }^{w}$, Planck18+BAO+BK15+IPTA $^{w}$, Planck18+BAO+BK15+FAST ${ }^{w}$, respectively 
The combinations of Planck18+BAO+BK15+FAST ${ }^{n}$ and Planck18+BAO+BK15+FAST ${ }^{w}$ effect the seven parameters slightly. The likelihood distributions for the tensor-to-scalar ratio change in Fig. 4. The constraint on parameter $r$ is

$r<0.049 \quad(95 \% \mathrm{CL})$

from Planck18+BAO+BK15+FAST datasets;

$r<0.049 \quad(95 \% \mathrm{CL})$

from Planck18+BAO+BK15+FAST ${ }^{n}$ datasets;

$r<0.049 \quad(95 \% \mathrm{CL})$

from Planck18+BAO+BK15+FAST ${ }^{w}$ datasets.

We also compare the narrow power spectrum and the wide power spectrum results in Figs. 5 and 6 from four datasets within LIGO, LISA, IPTA and FAST projects, respectively. The results show that the constraint from FAST have a significant impact on tensor-to-scalar ratio.

\section{Only consider the relic density of induced gravitational waves}

When we only consider the relic density of induced gravitational waves with respect to different frequencies from CMB scale to high frequencies including the range of LIGO and LISA, the numerical results are given in Fig. 7 which constrain the gravitational waves spectrum parameter space. The

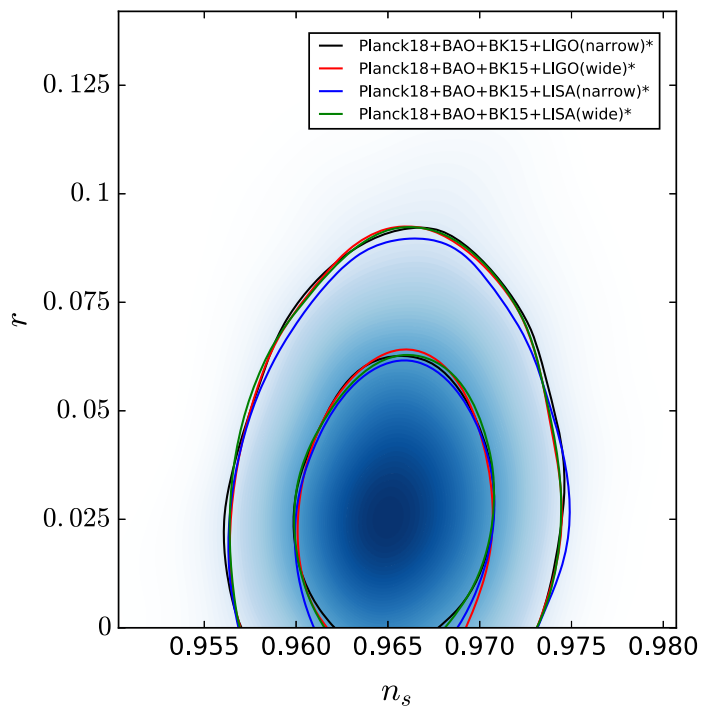

Fig. 7 The contour plot for the tensor-to-scalar ratio $r$ and parameter $n_{s}$ in the $\Lambda \mathrm{CDM}+r$ model at the $68 \%$ and $95 \% \mathrm{CL}$ from the combinations of Planck18+BAO+BK15+LIGO ${ }^{n *}$, Planck18+BAO+BK15+LIGO ${ }^{w *}$, Planck18+BAO+BK15+LISA ${ }^{n *}, \quad$ Planck18+BAO+BK15+LISA ${ }^{w *}$, respectively constraint on parameter $r$ is

$r<0.074 \quad(95 \% \mathrm{CL})$

from Planck18+BAO+BK15+LIGO ${ }^{n *}$ datasets;

$r<0.075(95 \% \mathrm{CL})$

from Planck18+BAO+BK15+LIGO ${ }^{w *}$ datasets;

$r<0.073(95 \% \mathrm{CL})$

from Planck18+BAO+BK15+LISA ${ }^{n *}$ datasets;

$r<0.076(95 \% \mathrm{CL})$

from Planck18+BAO+BK15+LISA ${ }^{w *}$ datasets. The results show that we calculate the relic of induced gravity at different frequencies to get more constraints on the cosmological parameters and explain the impact of Eqs. (7) and (8).

\section{Summary}

In the $\Lambda \mathrm{CDM}+r$ model, we constrain the scalar induced gravitational waves from the cosmic microwave background observations and the gravitational wave observations. The cosmological parameters are influenced by the evolution of the scalar induced gravitational waves from the additional scalar power spectrum, especially the combinations of Planck18+BAO+BK15+LISA. We also compare these numerical results from four datasets within LIGO, LISA, IPTA and FAST projects, respectively. The results show that FAST project has obvious impact on tensor-to-scalar ratio. Besides, we only consider the relic density of induced gravitational waves with respect to different frequencies from CMB scale to high frequencies including the range of LIGO and LISA.

Data Availability Statement This manuscript has associated data in a data repository. [Authors' comment: The data are public which can be obtained according references.]

Open Access This article is licensed under a Creative Commons Attribution 4.0 International License, which permits use, sharing, adaptation, distribution and reproduction in any medium or format, as long as you give appropriate credit to the original author(s) and the source, provide a link to the Creative Commons licence, and indicate if changes were made. The images or other third party material in this article are included in the article's Creative Commons licence, unless indicated otherwise in a credit line to the material. If material is not included in the article's Creative Commons licence and your intended use is not permitted by statutory regulation or exceeds the permitted use, you will need to obtain permission directly from the copyright holder. To view a copy of this licence, visit http://creativecomm ons.org/licenses/by/4.0/.

Funded by SCOAP 3 . 


\section{References}

1. E. Komatsu et al. (WMAP), Astrophys. J. Suppl. 180, 330-376 (2009). arXiv:0803.0547 [astro-ph]

2. N. Aghanim et al. (Planck), Astron. Astrophys. 641, A6 (2020). arXiv:1807.06209 [astro-ph.CO]

3. P.A.R. Ade et al. (BICEP2 and Keck Array Collaborations), Phys. Rev. Lett. 121, 221301 (2018). arXiv:1810.05216 [astro-ph.CO]

4. F. Beutler et al., Mon. Not. R. Astron. Soc. 416, 3017 (2011). arXiv:1106.3366 [astro-ph.CO]

5. A.J. Ross, L. Samushia, C. Howlett, W.J. Percival, A. Burden, M. Manera, Mon. Not. R. Astron. Soc. 449(1), 835 (2015). arXiv:1409.3242 [astro-ph.CO]

6. S. Alam et al. (BOSS), Mon. Not. R. Astron. Soc. 470(3), 26172652 (2017). arXiv:1607.03155 [astro-ph.CO]

7. K. Tomita, Prog. Theor. Phys. 37, 831 (1967)

8. C. Yuan, Z.C. Chen, Q.G. Huang, Phys. Rev. D 101(4), 043019 (2020). arXiv:1910.09099 [astro-ph.CO]

9. C. Yuan, Z.C. Chen, Q.G. Huang, Phys. Rev. D 100(8), 081301 (2019). arXiv:1906.11549 [astro-ph.CO]

10. C. Yuan, Q.G. Huang, arXiv:2007.10686 [astro-ph.CO]

11. C. Yuan, Z.C. Chen, Q.G. Huang, Phys. Rev. D 101(6), 063018 (2020). arXiv:1912.00885 [astro-ph.CO]

12. K. Nakamura, arXiv:1912.12805 [gr-qc]

13. G. Domènech, M. Sasaki, Phys. Rev. D 103(6), 063531 (2021). arXiv:2012.14016 [gr-qc]

14. J.C. Hwang, D. Jeong, H. Noh, Astrophys. J. 842(1), 46 (2017). arXiv:1704.03500 [astro-ph.CO]

15. K. Inomata, T. Terada, Phys. Rev. D 101(2), 023523 (2020). arXiv: 1912.00785 [gr-qc]

16. K. Tomikawa, T. Kobayashi, Phys. Rev. D 101(8), 083529 (2020). arXiv:1910.01880 [gr-qc]

17. S. Matarrese, S. Mollerach, M. Bruni, Phys. Rev. D 58, 043504 (1998). arXiv:astro-ph/9707278

18. G. Domènech, Int. J. Mod. Phys. D 29(03), 2050028 (2020). arXiv:1912.05583 [gr-qc]

19. L. Alabidi, K. Kohri, M. Sasaki, Y. Sendouda, JCAP 09, 017 (2012). arXiv:1203.4663 [astro-ph.CO]

20. L. Alabidi, K. Kohri, M. Sasaki, Y. Sendouda, JCAP 05, 033 (2013). arXiv:1303.4519 [astro-ph.CO]

21. H. Assadullahi, D. Wands, Phys. Rev. D 81, 023527 (2010). arXiv:0907.4073 [astro-ph.CO]

22. K. Inomata, K. Kohri, T. Nakama, T. Terada, JCAP 10, 071 (2019). arXiv:1904.12878 [astro-ph.CO]
23. K. Inomata, K. Kohri, T. Nakama, T. Terada, Phys. Rev. D 100(4), 043532 (2019). arXiv:1904.12879 [astro-ph.CO]

24. Z. Yi, Y. Gong, B. Wang, Z.H. Zhu, Phys. Rev. D 103(6), 063535 (2021). arXiv:2007.09957 [gr-qc]

25. J.R. Espinosa, D. Racco, A. Riotto, JCAP 09, 012 (2018). arXiv:1804.07732 [hep-ph]]

26. K. Kohri, T. Terada, Phys. Rev. D 97(12), 123532 (2018). arXiv: 1804.08577 [gr-qc]

27. K.N. Ananda, C. Clarkson, D. Wands, Phys. Rev. D 75, 123518 (2007). arXiv:gr-qc/0612013

28. G. Domènech, S. Pi, M. Sasaki, JCAP 08, 017 (2020). arXiv:2005.12314 [gr-qc]

29. F. Hajkarim, J. Schaffner-Bielich, Phys. Rev. D 101(4), 043522 (2020). arXiv:1910.12357 [hep-ph]

30. K. Inomata, T. Nakama, Phys. Rev. D 99(4), 043511 (2019). arXiv:1812.00674 [astro-ph.CO]

31. Y. Lu, Y. Gong, Z. Yi, F. Zhang, JCAP 12, 031 (2019). arXiv: 1907.11896 [gr-qc]

32. S. Pi, M. Sasaki, JCAP 09, 037 (2020). arXiv:2005.12306 [gr-qc]

33. C. Fu, P. Wu, H. Yu, Phys. Rev. D 101(2), 023529 (2020). arXiv:1912.05927 [astro-ph.CO]

34. B.P. Abbott et al. (LIGO Scientific and Virgo Collaborations), Phys. Rev. Lett. 118(12), 121101 (2017). arXiv:1612.02029 [gr-qc]

35. B.P. Abbott et al. (LIGO Scientific and Virgo Collaborations), arXiv: 1903.02886 [gr-qc]

36. E. Thrane, J.D. Romano, Phys. Rev. D 88(12), 124032 (2013). arXiv: 1310.5300 [astro-ph.IM]

37. C. Caprini et al., JCAP 1604(04), 001 (2016). arXiv:1512.06239 [astro-ph.CO]

38. R.W. Hellings, G.S. Downs, Astrophys. J. 265, L39 (1983)

39. J.P.W. Verbiest et al., Mon. Not. R. Astron. Soc. 458(2), 1267 (2016). arXiv: 1602.03640 [astro-ph.IM]

40. R. Nan et al., Int. J. Mod. Phys. D 20, 989 (2011). arXiv:1105.3794 [astro-ph.IM]

41. K. Kuroda, W.T. Ni, W.P. Pan, Int. J. Mod. Phys. D 24(14), 1530031 (2015). arXiv:1511.00231 [gr-qc]

42. J. Li, Z.C. Chen, Q.G. Huang, Sci. China Phys. Mech. Astron. 62(11), 110421 (2019). arXiv:1907.09794 [astro-ph.CO]

43. J. Li, G.H. Guo, arXiv:2101.07970 [astro-ph.CO]

44. J. Li, Q.G. Huang, Eur. Phys. J. C 78(11), 980 (2018). arXiv:1806.01440 [astro-ph.CO]

45. A. Lewis, S. Bridle, Phys. Rev. D 66, 103511 (2002). arXiv:astro-ph/0205436 